\title{
Decaying Two-Dimensional Turbulence in a Circular Container
}

\author{
Kai Schneider* \\ LMSNM-CNRS \& CMI, Université de Provence, Marseille, France \\ Marie Farge $e^{\dagger}$ \\ LMD-CNRS, Ecole Normale Supérieure, Paris, France \\ (Received 28 April 2005; published 8 December 2005)
}

\begin{abstract}
We present direct numerical simulations of two-dimensional decaying turbulence at initial Reynolds number $5 \times 10^{4}$ in a circular container with no-slip boundary conditions. Starting with random initial conditions the flow rapidly exhibits self-organization into coherent vortices. We study their formation and the role of the viscous boundary layer on the production and decay of integral quantities. The no-slip wall produces vortices which are injected into the bulk flow and tend to compensate the enstrophy dissipation. The self-organization of the flow is reflected by the transition of the initially Gaussian vorticity probability density function (PDF) towards a distribution with exponential tails. Because of the presence of coherent vortices the pressure PDF become strongly skewed with exponential tails for negative values.
\end{abstract}

Two-dimensional turbulence in bounded domains plays an important role in oceanography, e.g., in vortex formation in coastal currents. Many experiments in rotating tanks, e.g., in Ref. [1], resulting in quasi-two-dimensional geostrophic flows, have shown the formation of long-lived coherent vortices. In contrast, only a few numerical studies of 2D turbulence in bounded circular domains have been performed so far. Some numerical simulations of decaying 2D turbulence in a circular domain with no-slip boundary conditions have been presented in Refs. [2-4], which used a spectral method with Bessel functions of the first kind, i.e., circular analogues of the Chandrasekhar-Reid functions. Because of the numerical complexity of the spectral scheme these simulations were limited to low resolution. Therefore only flows at low Reynolds numbers have thus been studied, i.e., $\operatorname{Re}<10^{3}$, where $\operatorname{Re}$ is based on the rms initial velocity and the circle radius. Numerical simulations of forced 2D turbulence in circular geometry for Reynolds numbers up to 3500 using a Tchebycheff-Fourier discretization have been presented in Ref. [5].

The aim of this Letter is to present direct numerical simulation of two-dimensional decaying turbulence in a circular geometry with higher initial Reynolds number of $5 \times 10^{4}$ computed at resolution $N=1024^{2}$. The numerical scheme is based on a Fourier pseudospectral method with semi-implicit time discretization and adaptive time stepping [6]. The Navier-Stokes equations are solved in a square domain of size $L=2 \pi$ using the vorticity-velocity formulation. The circular container $\Omega$ of radius $R=2.8$ is thus imbedded in the square domain and the no-slip boundary conditions on the wall $\partial \Omega$ are imposed using a volume penalization method. A mathematical analysis of the method is given in Ref. [7], proving its convergence towards the Navier-Stokes equations with no-slip boundary conditions. Its numerical validation can be found in Refs. $[6,8]$.
The resulting governing equation is

$$
\partial_{t} \omega+\vec{u} \cdot \vec{\nabla} \omega-\nu \nabla^{2} \omega+\vec{\nabla} \times\left(\frac{1}{\eta} \chi \vec{u}\right)=0
$$

where $\vec{u}$ is the divergence-free velocity field, i.e., $\vec{\nabla} \cdot \vec{u}=$ $0, \omega=\vec{\nabla} \times \vec{u}$ the vorticity, $\nu$ the kinematic viscosity, and $\chi(\vec{x})$ a mask function which is 0 inside the fluid, i.e., $\vec{x} \in$ $\Omega$, and 1 inside the solid wall. The penalization parameter $\eta$ is chosen to be sufficiently small $\left(\eta=10^{-3}\right)$ [6].

Different invariants of the flow, i.e., quantities which are conserved by the flow dynamics for inviscid flows, can be derived [9]: (i) the circulation $\Gamma$ (total vorticity) is defined as

$$
\Gamma=\int_{\Omega} \omega d \vec{x}=\oint_{\partial \Omega} \vec{u} \cdot d s ;
$$

(ii) energy $E$, enstrophy $Z$, and palinstrophy $P$ as

$$
\begin{gathered}
E=\frac{1}{2} \int_{\Omega}|\vec{u}|^{2} d \vec{x}, \quad Z=\frac{1}{2} \int_{\Omega}|\omega|^{2} d \vec{x}, \\
P=\frac{1}{2} \int_{\Omega}|\vec{\nabla} \omega|^{2} d \vec{x},
\end{gathered}
$$

respectively. (iii) the energy dissipation is given by $d_{t} E=$ $-2 \nu Z$ and the enstrophy dissipation by

$$
d_{t} Z=-2 \nu P+\nu \oint_{\partial \Omega} \omega(\vec{n} \cdot \vec{\nabla} \omega) d s,
$$

where $\vec{n}$ denotes the outer normal vector with respect to $\partial \Omega$. The surface integral reflects the enstrophy production at the wall involving the vorticity and its gradients. (iv) the angular momentum $M$ of the flow with respect to the center of the domain is

$$
M=2 \int_{\Omega} \psi d \vec{x}
$$

where $\psi=\nabla^{-2} \omega$ denotes the stream function. 

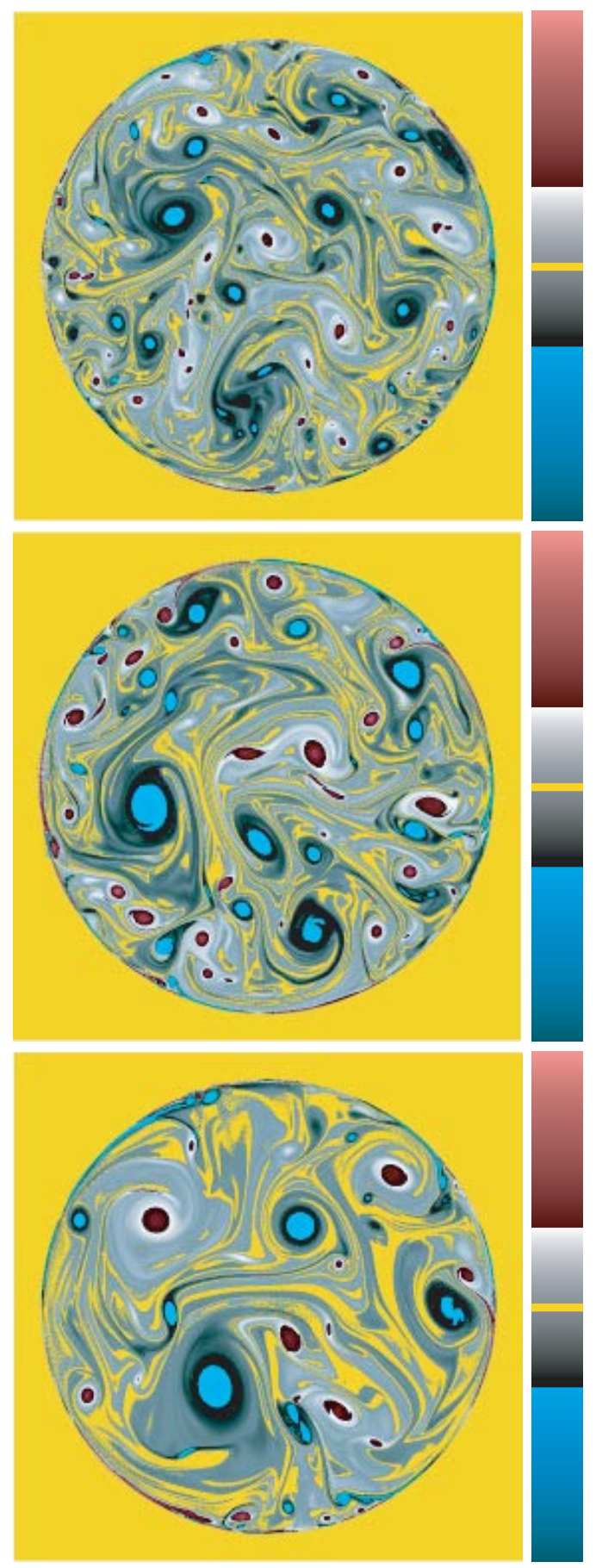

FIG. 1 (color). Vorticity fields at $\tau=80,160$, and 320 .

As initial conditions we choose a correlated Gaussian noise with zero angular momentum and an energy spectrum $E(k) \propto k^{-4}$. The initial Reynolds number is $\mathrm{Re}=$ $2 R \sqrt{2 E} / \nu=5 \times 10^{4}$. We introduce a dimensionless time $\tau=t / t_{e}$ based on the initial eddy turnover time $t_{e}=$ $1 / \sqrt{2 Z(0)}=0.061$. The flow has been integrated for $650 t_{e}$ corresponding to more than $10^{5}$ time steps.

Figure 1 shows snapshots of the vorticity field at $\tau=80$, 160 , and 320 . We observe the formation of vorticity sheets

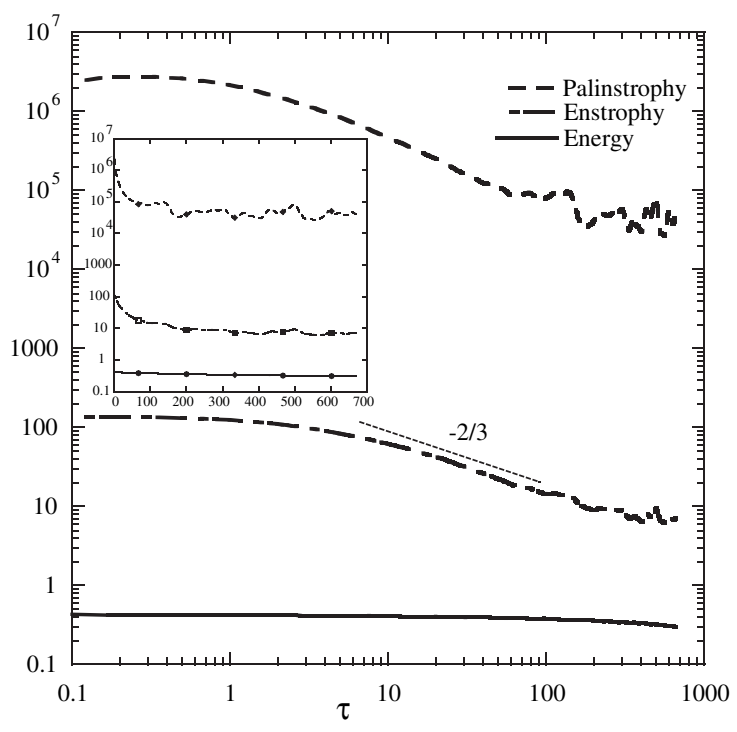

FIG. 2. Time evolution of energy $E$, enstrophy $Z$, and palinstrophy $P$ in log-log coordinates (Inset: time evolution in log-lin coordinates).

at the wall which roll up into coherent vortices. This active unstable strong boundary layer persists throughout the simulation. The resulting continuous injection of vorticity and vorticity gradients into the flow leads to a concomitant increase of the energy dissipation. Where the boundary layer detaches from the wall we observe the formation of dipolar vortices, which then move into the bulk flow and interact with other vortices as observed in rotating tanks [1].

In Fig. 2 we plot the time evolution of different integral quantities. We observe that the kinetic energy slowly decays. At the final instant the energy has lost $71 \%$ of its initial value, while the enstrophy has decreased to only

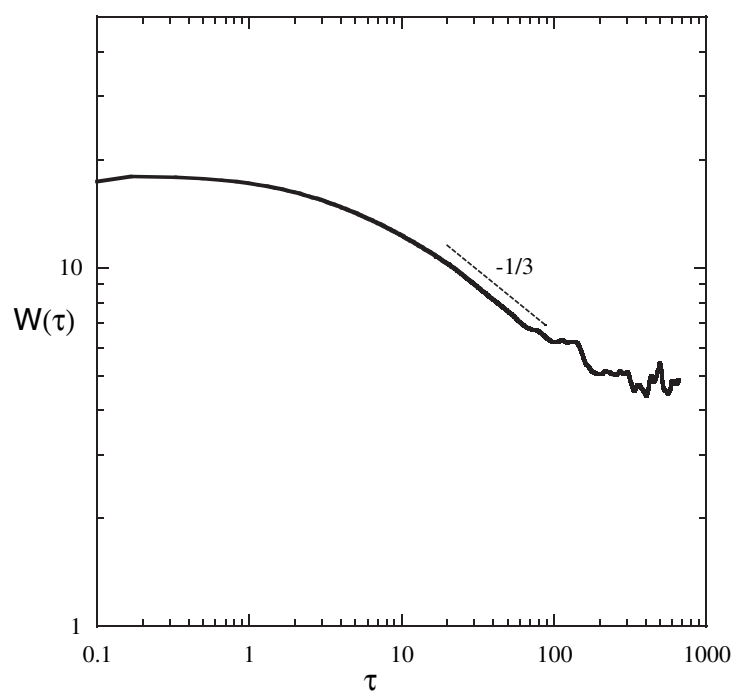

FIG. 3. Time evolution of the average wave number $W=$ $\sqrt{Z / E}$. 


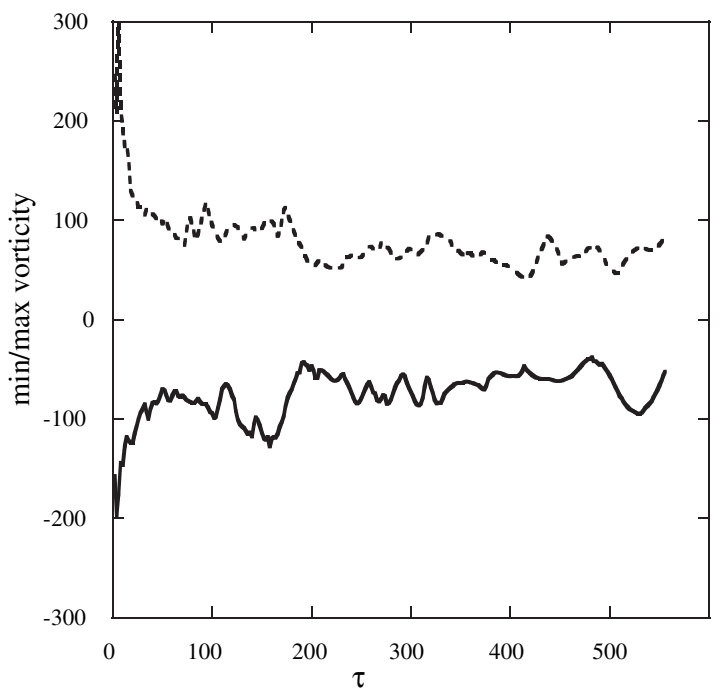

FIG. 4. Time evolution of the maximum and minimum vorticity $\omega$.

$5.1 \%$ and the palinstrophy to only $1.5 \%$ of their initial values. The enstrophy exhibits a self-similar decay over one decade (from $\tau=10$ to about 100), proportional to $t^{-2 / 3}$. Note that this is much slower than in double periodic simulations [10] where typically a slope of -1 is observed for the enstrophy decay. At later times, for $\tau>150$, we also observe a nonmonotonous behavior for $Z$ and $P$ which is due to the generation of vorticity and its gradients at the no-slip wall. The inset, which shows the time evolution of $E, Z$, and $P$ in $\log$-lin coordinates, illustrates that, after a transition phase up to $\tau=150$, the palinstrophy and enstrophy decay is strongly reduced.

The mean square wave number $W=\sqrt{Z / E}$, which is inversely proportional to the Taylor microscale, is bounded

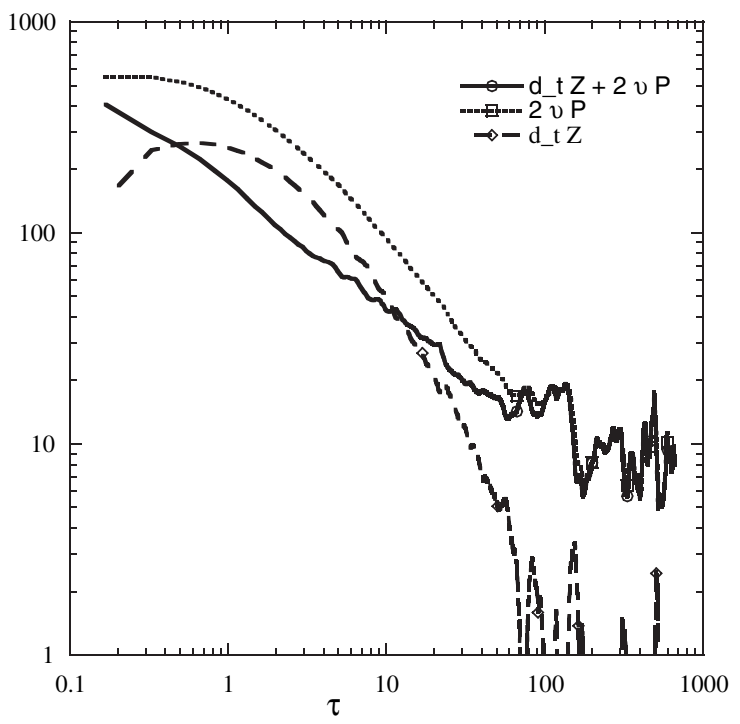

FIG. 5. Time evolution $d_{t} Z, 2 \nu P$, and $\nu \oint_{\partial \Omega} \omega(\vec{n} \cdot \vec{\nabla} \omega) d s=$ $d_{t} Z+2 \nu P$.

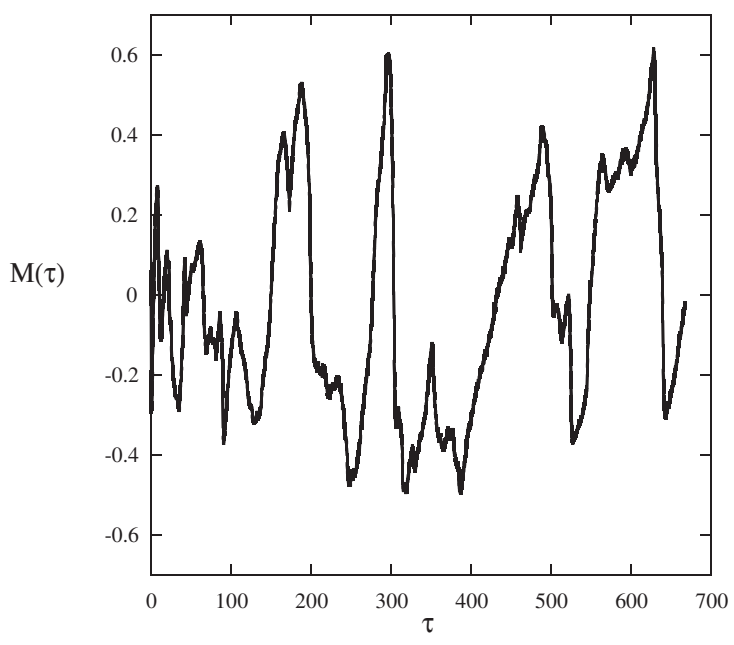

FIG. 6. Time evolution of angular momentum $M$.

from below by one, corresponding to structures of the size of the domain. For unbounded flows one can show that $d_{t}(W)^{2} \leq 0$; i.e., the average vortex size is monotonously increasing [10]. In the present case we observe a monotonous decay up to $\tau=100$. At later times a nonmonotonous behavior is found which is due to the intermittent generation of vortices at the no-slip wall (cf. Fig. 3).

The time evolution of minimum and maximum vorticity (Fig. 4) also exhibits an oscillating behavior, which is in contrast to 2D decaying turbulence without walls, where the maximum of $|\omega|$ is decaying monotonously. Note that the circulation $\Gamma$ (not shown here) almost vanishes with a rms value below $3.4 \times 10^{-6}$.

Figure 5 illustrates the different terms in Eq. (3) for the enstrophy dissipation. We observe a monotonous decay of all terms up to $\tau=100$. The enstrophy production term at the wall yields a power-law behavior with slope $-2 / 3$, and for later times oscillations can be observed. Furthermore, the enstrophy production at the wall $\left(\nu \oint_{\partial \Omega} \omega(\vec{n} \cdot \vec{\nabla} \omega) d s\right)$

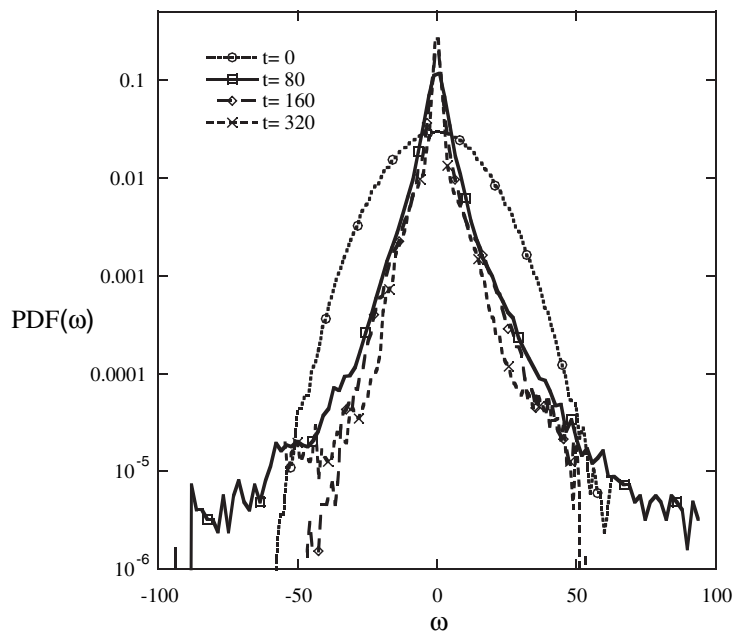

FIG. 7. PDF of vorticity $\tau=0,80,160$, and 320 . 
TABLE I. Statistical properties of the vorticity field. Variance, skewness, flatness at $\tau=0,80,160$, and 320 .

\begin{tabular}{lccr}
\hline \hline Vorticity & Variance & Skewness & Flatness \\
\hline$\omega(\tau=0)$ & 183.95 & -0.02 & 3.00 \\
$\omega(\tau=80)$ & 33.01 & 0.04 & 23.04 \\
$\omega(\tau=160)$ & 20.97 & 0.71 & 15.11 \\
$\omega(\tau=320)$ & 14.75 & 0.32 & 25.98 \\
\hline \hline
\end{tabular}

coincides with the term $\nu P$ for $\tau>100$. This implies that the enstrophy dissipation $d_{t} Z$ becomes negligible and oscillates around zero. In Fig. 6 we plot the time evolution of angular momentum $M(t)$, normalized with the angular momentum of a flow in solid body rotation having the same energy $E(t)$. We observe alternating periods of positive and negative angular momentum, with a sawtooth behavior where rises are followed by abrupt decays. This is in contrast to the spontaneous spin-up encountered in decaying turbulent flows in square domains with no-slip boundary conditions [11]. This confirms the conjecture, that spin-up is likely to be absent in circular geometries [3].

Figure 7 shows the probability density functions (PDF)s of vorticity at different time instants. Starting with a Gaussian shape at $\tau=0$ the vorticity PDF becomes more and more non-Gaussian with heavy tails, although its symmetry is preserved (Table I). This confirms that the flow intermittency is due to the formation of vortex sheets and the resulting coherent vortices.

The PDF of pressure, which is initially almost Gaussian, becomes strongly skewed (Table II) and exhibits exponential tails proportional to $\exp (10 p)$ for negative values (Fig. 8). The much stronger probability for negative values and the resulting skewness is a signature of coherent vortices.

In conclusion, we have shown, by means of direct numerical simulation performed in cylindrical geometry, that no-slip boundaries play a crucial role for decaying turbulent flows. At early times we observe a decay of the flow which leads to self-organization and the emergence of vortices in the bulk flow, similarly to flows in double periodic boxes. At later times, the production of coherent vortices at the boundary compensates the enstrophy dissipation and the flow decay is drastically reduced. This is

TABLE II. Statistical properties of the pressure field. Variance, skewness, flatness at $\tau=0,80,160$, and 320 .

\begin{tabular}{lccr}
\hline \hline Pressure & Variance & Skewness & Flatness \\
\hline$p(\tau=0)$ & $1.66 \times 10^{-2}$ & -0.49 & 3.59 \\
$p(\tau=80)$ & $4.35 \times 10^{-2}$ & -2.35 & 9.32 \\
$p(\tau=160)$ & $4.48 \times 10^{-2}$ & -2.86 & 12.40 \\
$p(\tau=320)$ & $5.21 \times 10^{-2}$ & -2.96 & 12.86 \\
\hline \hline
\end{tabular}

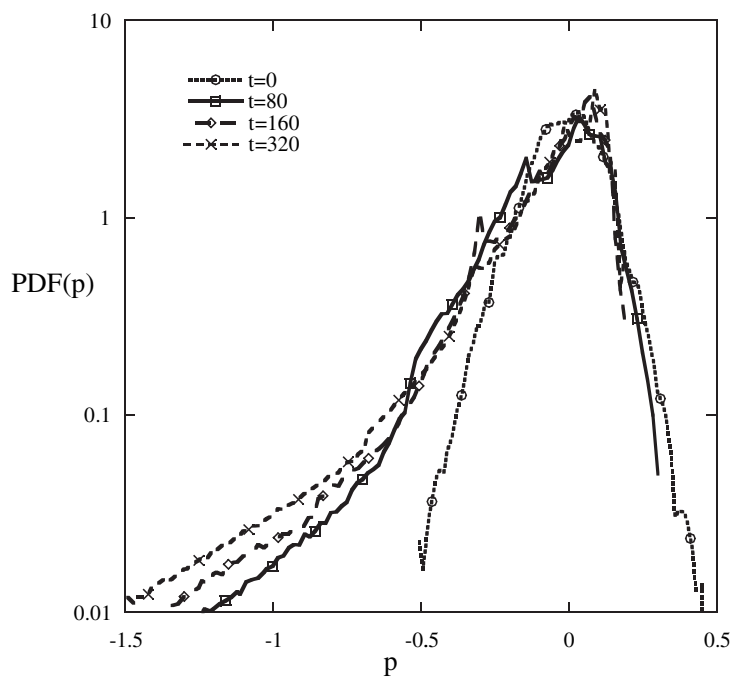

FIG. 8. PDF of pressure $\tau=0,80,160$, and 320 .

reflected in the time evolution of enstrophy and palinstrophy which decay in a nonmonotonous way. The pressure PDF is strongly skewed with an exponential shape for negative values due to the presence of coherent vortices. The present simulation at initial $\mathrm{Re}=5 \times 10^{4}$ confirms that spontaneous spin-up seems to be absent for circular geometries with no-slip walls as conjectured in Refs. [2,5].

We thankfully acknowledge financial support from the European Union project IHP on "Breaking complexity" and from the contract CEA/EURATOM No. V.3258.001.

*Electronic address: kschneid@cmi.univ-mrs.fr

${ }^{\dagger}$ Electronic address: farge@1md.ens.fr

[1] J. Aubert, S. Jung, and H.L. Swinney, Geophys. Res. Lett. 29, 1876 (2002).

[2] S. Li and D. Montgomery, Phys. Lett. A 218, 281 (1996).

[3] S. Li, D. Montgomery, and W. B. Jones, J. Plasma Phys. 56, 615 (1996).

[4] S. Li, D. Montgomery, and W. B. Jones, Theor. Comput. Fluid Dyn. 9, 167 (1997).

[5] H. J.H. Clercx, A.H. Nielsen, D. J. Torres, and E. A. Coutsias, Eur. J. Mech. B, Fluids 20, 557 (2001).

[6] K. Schneider, Comput. Fluids 34, 1223 (2005).

[7] P. Angot, C. H. Bruneau, and P. Fabrie, Numer. Math. 81, 497 (1999).

[8] N. Kevlahan and J.-M. Ghidaglia, Eur. J. Mech. B, Fluids 20, 333 (2001).

[9] R. H. Kraichnan and D. Montgomery, Rep. Prog. Phys. 43, 547 (1980).

[10] W.H. Matthaeus, W. T. Stribling, D. Martinez, S. Oughton, and D. Montgomery, Phys. Rev. Lett. 66, 2731 (1991).

[11] H. J.H. Clercx, S. R. Maassen, and G. J.F. van Heijst, Phys. Rev. Lett. 80, 5129 (1998). 